Supporting information

\title{
High Yield Organic Superconductors via Solution-Phase Alkali Metal Doping at Room Temperature
}

\author{
Taekyung Yoon ${ }^{1}$, Jin Young $\mathrm{Koo}^{1}$, Hee Cheul Choi ${ }^{1 *}$ \\ ${ }^{1}$ Department of Chemistry, Pohang University of Science and Technology (POSTECH), Pohang 37673, Republic of \\ Korea \\ *E-mail: choihc@postech.edu
}

This PDF file includes:

Figure S1 to S8

Table S1 to S3 
Table of Contents

1. Field dependence of magnetization of $\mathrm{K}_{3} \mathrm{C}_{60} \quad$ P 3

2. Temperature and field dependence of magnetization of $\mathrm{Rb}_{3} \mathrm{C}_{60} \quad P 4$

3. Raman spectra of pristine $\mathrm{C}_{60}$ and solution-phase-doped $\mathrm{K}_{3} \mathrm{C}_{60} \quad$ P 5

4. $M / H$ versus $T$ of $\mathrm{K}_{3} \mathrm{C}_{60}$ prepared by $\mathrm{SPD} / \mathrm{AM}$ depending on annealing time $\quad$ P 6

5. $M / H$ versus $T$ of $\mathrm{K}_{3} \mathrm{C}_{60}$ prepared by SPD/MA depending on annealing time $\quad$ P 7

6. $M / H$ versus $T$ of $\mathrm{K}_{3} \mathrm{C}_{60}$ prepared by SPD/THF depending on annealing time $\quad$ P 8

7. $\mathrm{X}$-ray diffraction patterns for non-annealed and annealed $\mathrm{K}_{3} \mathrm{C}_{60} \quad$ P 9

8. X-ray diffraction patterns for pristine $\mathrm{C}_{60}$ and solution-phase-doped $\mathrm{K}_{3} \mathrm{C}_{60} \quad P 10$

9. Spin amount of $\mathrm{K}_{\mathrm{x}} \mathrm{C}_{60}(\mathrm{x}=0,1,2,3)$ solutions $\quad$ P 11

10. Shielding fraction of solution-phase-doped $\mathrm{K}_{3} \mathrm{C}_{60} \quad P 12$

11. Assigned crystal information of solution-phase-doped $\mathrm{K}_{3} \mathrm{C}_{60} \quad P 13$ 

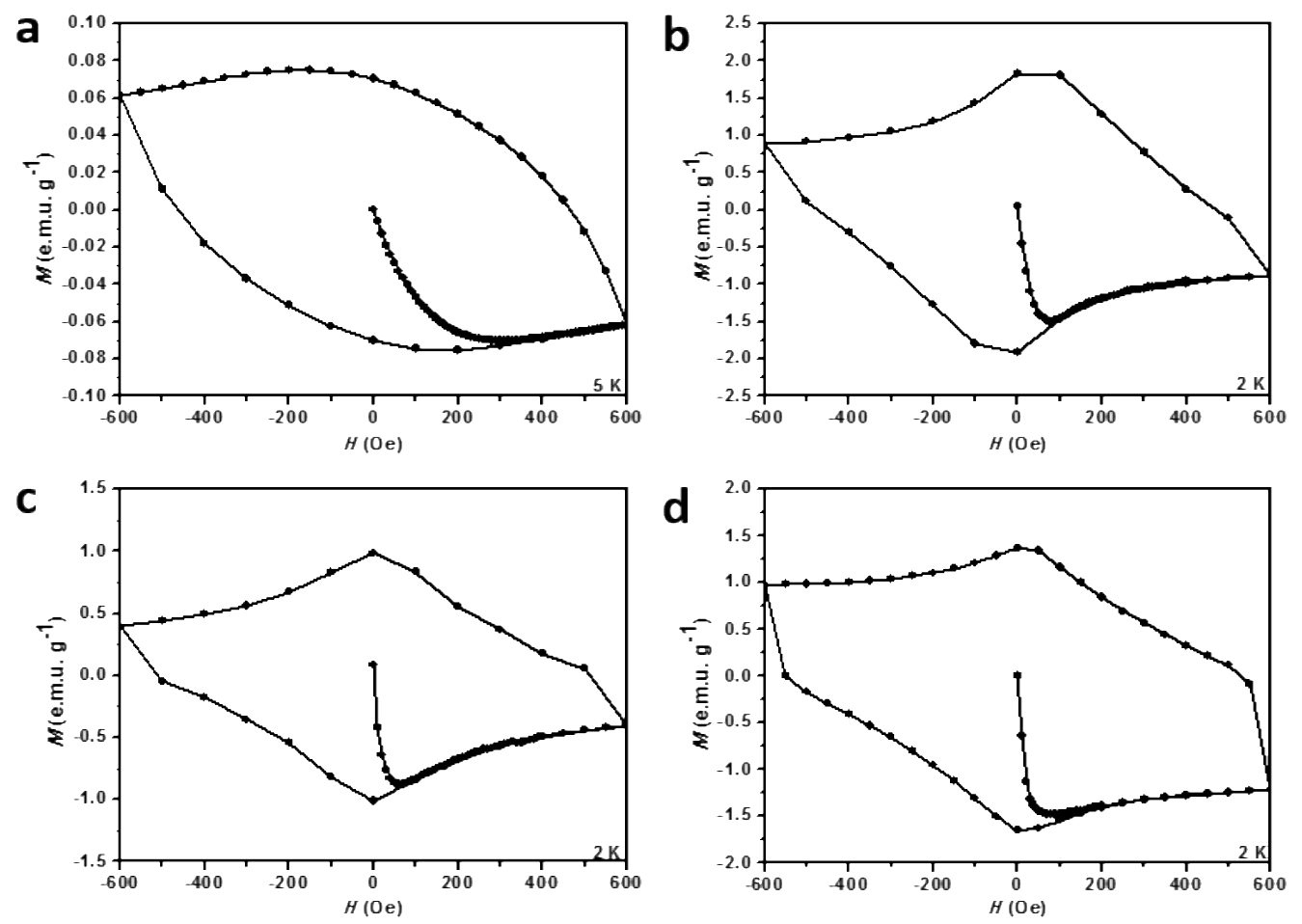

Figure S1. Full information of field dependence of magnetization of $\mathrm{K}_{3} \mathrm{C}_{60}$. (a) $\mathrm{K}_{3} \mathrm{C}_{60}$ prepared by conventional vapor phase doping, measured at $5 \mathrm{~K}$. (b) (d) $\mathrm{K}_{3} \mathrm{C}_{60}$ prepared by SPD methods, measured at $2 \mathrm{~K}$, using (b) SPD/AM, (c) SPD/MA, (d) SPD/THF. 

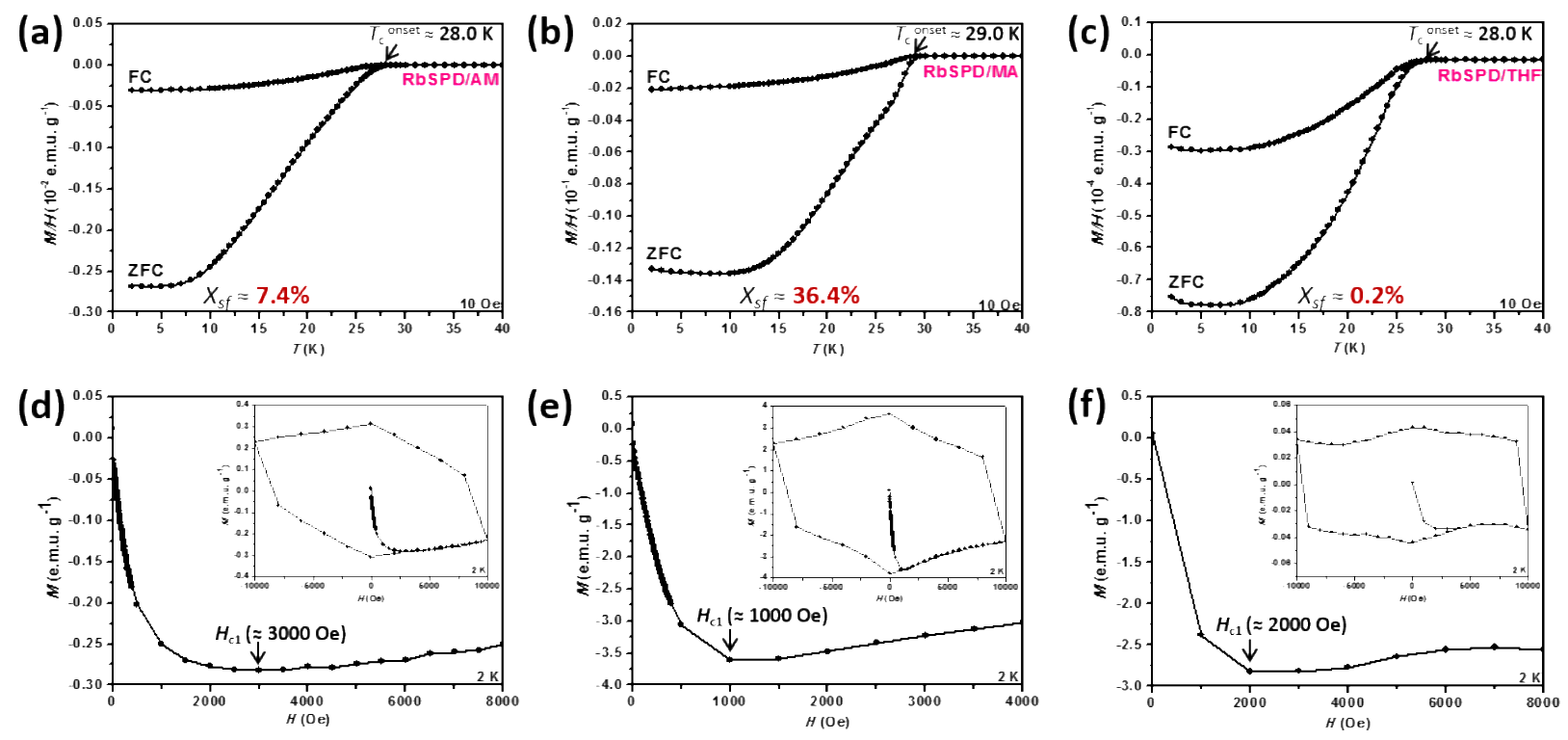

Figure S2. Temperature dependence of magnetization $(M / H$ versus $T)$ and field dependence of magnetization ( $M$ versus $H$ ) plot of $\mathrm{Rb}_{3} \mathrm{C}_{60}$ prepared by (a, d) SPD/AM, (b, e) SPD/MA, and (c, f) SPD/THF methods. The shielding fraction is estimated with their magnetization at $2 \mathrm{~K}$. The field dependent magnetization is measured at $2 \mathrm{~K}$. Each lower critical field value $\left(H_{\mathrm{cl}}\right)$ is marked with an arrow. Each inset shows full information of $M$ versus $H$ plot. 


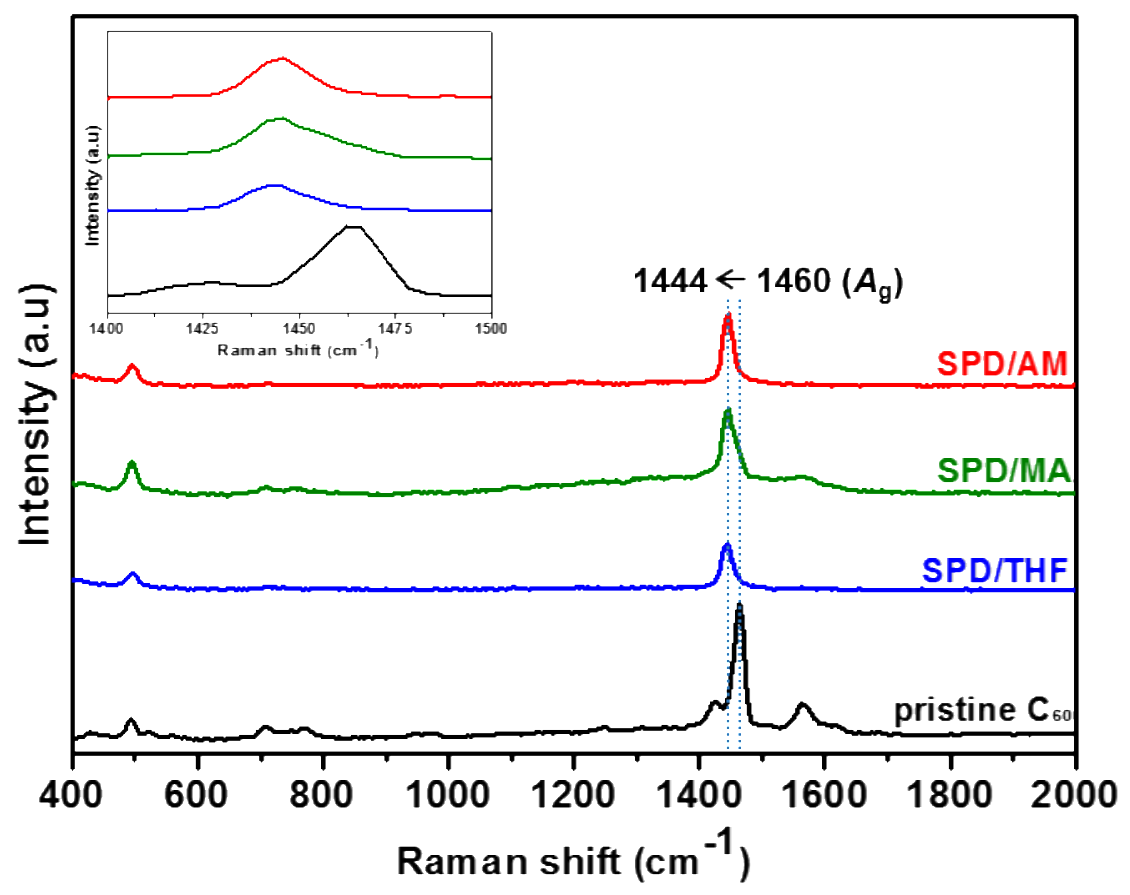

Figure S3. Raman spectra of $\mathrm{K}_{3} \mathrm{C}_{60}$ prepared by SPD method. SPD/AM (red), SPD/MA (green), $\mathrm{SPD} / \mathrm{THF}$ (blue) and pristine $\mathrm{C}_{60}$ (black). A peak corresponding to $A_{\mathrm{g}}$ vibrational mode shows a distinct downshift. 

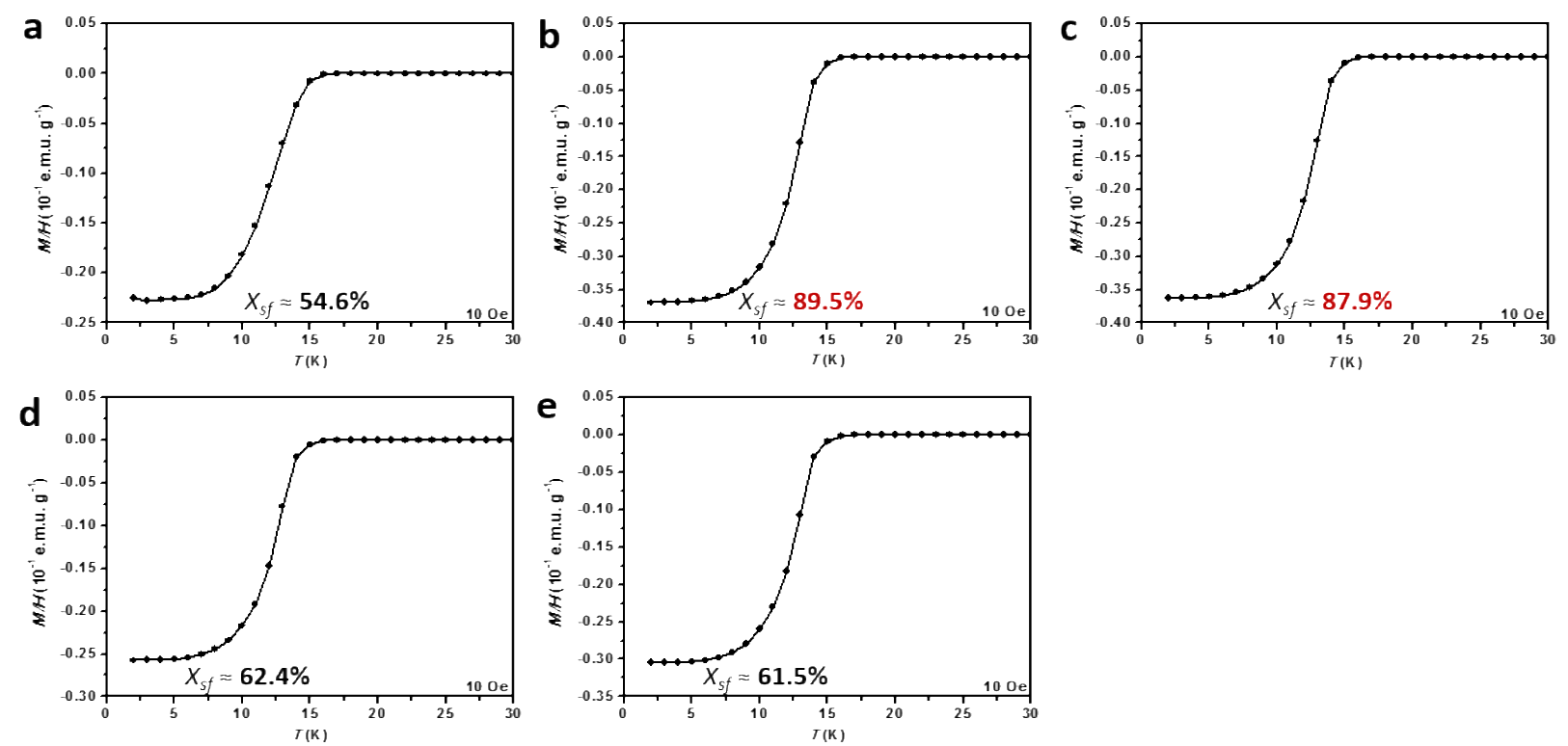

Figure S4. ZFC data of $M / H$ versus $T$ plots of $\mathrm{K}_{3} \mathrm{C}_{60}$ prepared by SPD/AM as a function of annealing time (measured at $2 \mathrm{~K}$ ). (a) $24 \mathrm{~h}$, (b) $30 \mathrm{~h}$, (c) $36 \mathrm{~h}$, (d) $42 \mathrm{~h}$, (e) $48 \mathrm{~h}$. 

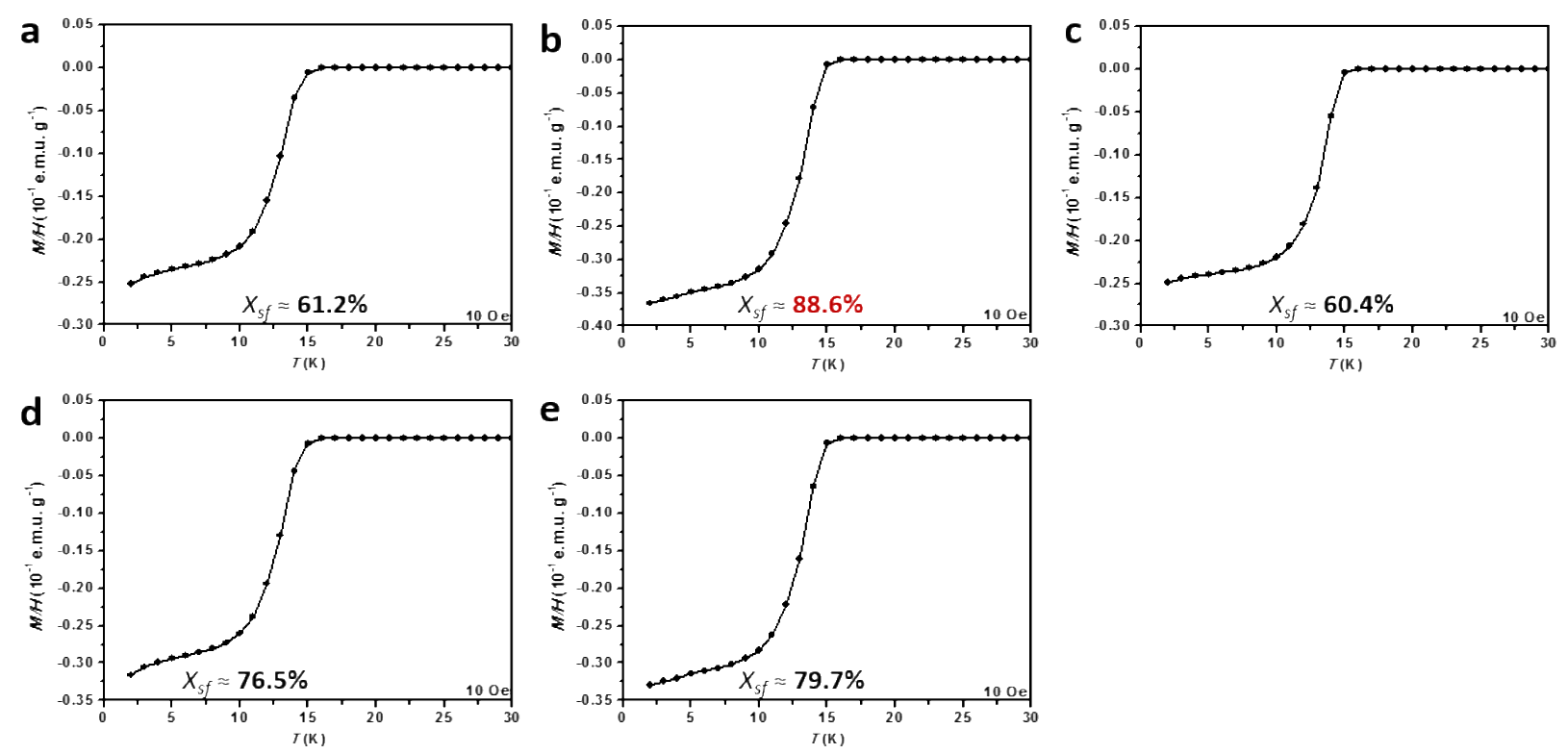

Figure S5. ZFC data of $M / H$ versus $T$ plots of $\mathrm{K}_{3} \mathrm{C}_{60}$ prepared by SPD/MA as a function of annealing time (measured at $2 \mathrm{~K}$ ). (a) $24 \mathrm{~h}$, (b) $30 \mathrm{~h}$, (c) $36 \mathrm{~h}$, (d) $42 \mathrm{~h}$, (e) $48 \mathrm{~h}$. 

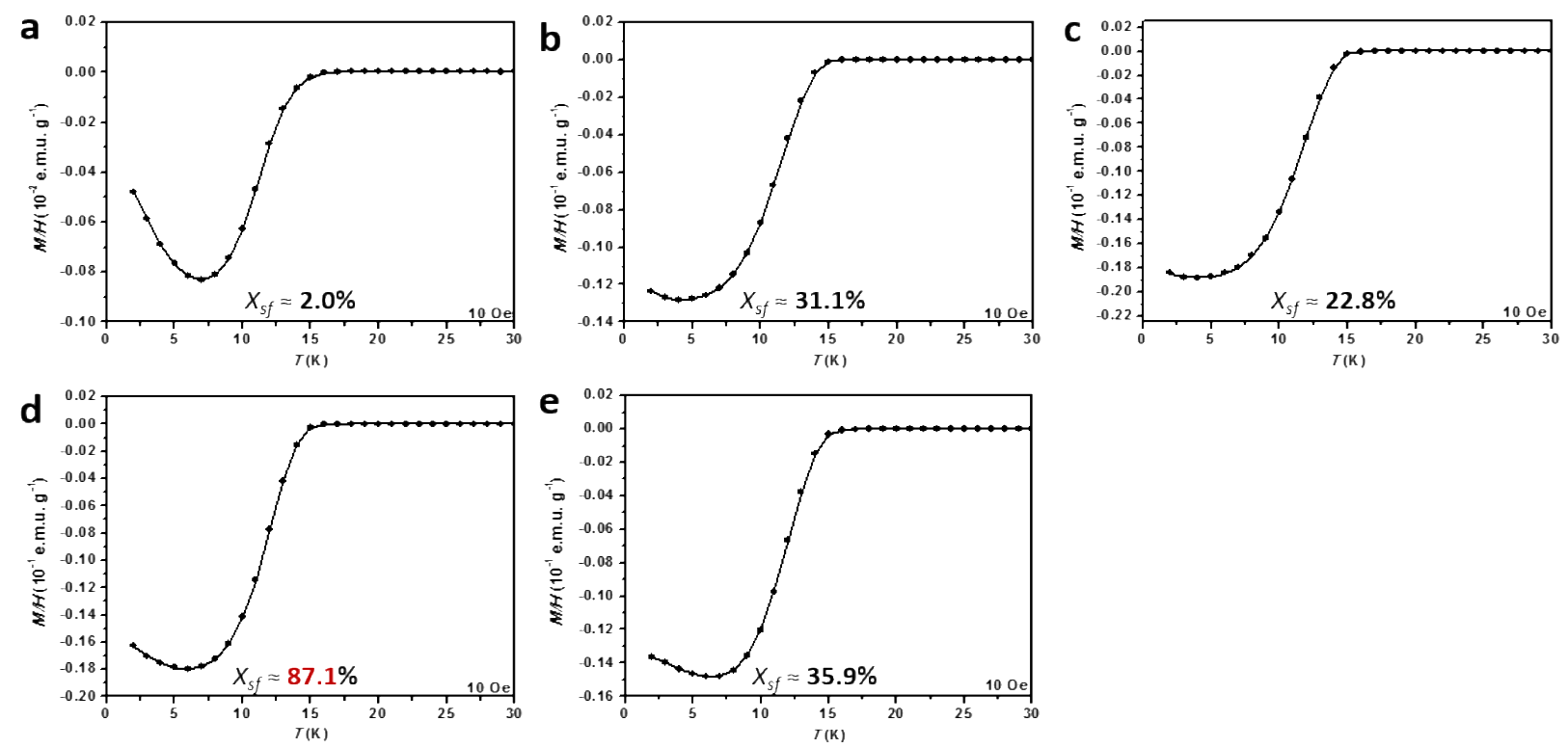

Figure S6. ZFC data of $M / H$ versus $T$ plots of $\mathrm{K}_{3} \mathrm{C}_{60}$ prepared by SPD/THF as a function of annealing time (measured at $2 \mathrm{~K}$ ). (a) $24 \mathrm{~h}$, (b) $30 \mathrm{~h}$, (c) $36 \mathrm{~h}$, (d) $42 \mathrm{~h}$, (e) $48 \mathrm{~h}$. 

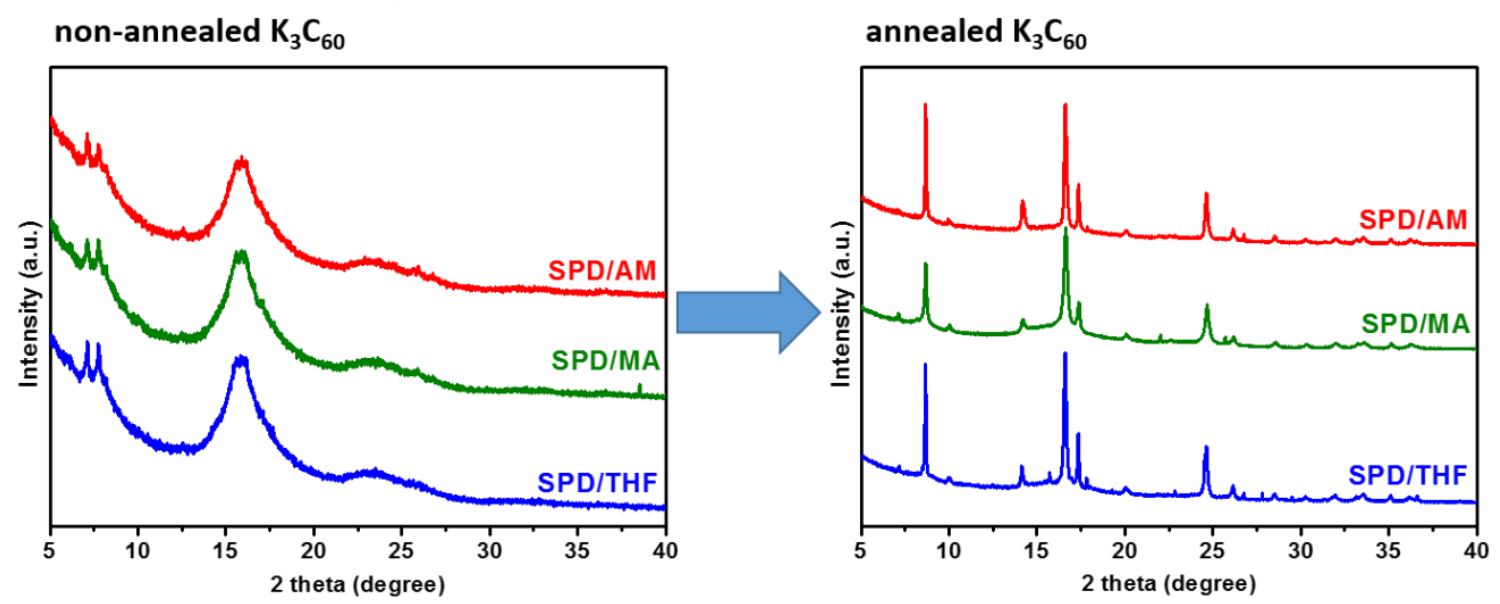

Figure S7. X-ray diffraction patterns for non-annealed (left), and annealed (right) $\mathrm{K}_{3} \mathrm{C}_{60}$. Annealing condition was $200{ }^{\circ} \mathrm{C}$ in argon-filled condition for $36 \mathrm{~h}$ (SPD/AM), $30 \mathrm{~h}$ (SPD/MA), $42 \mathrm{~h}(\mathrm{SPD} / \mathrm{THF})$. 

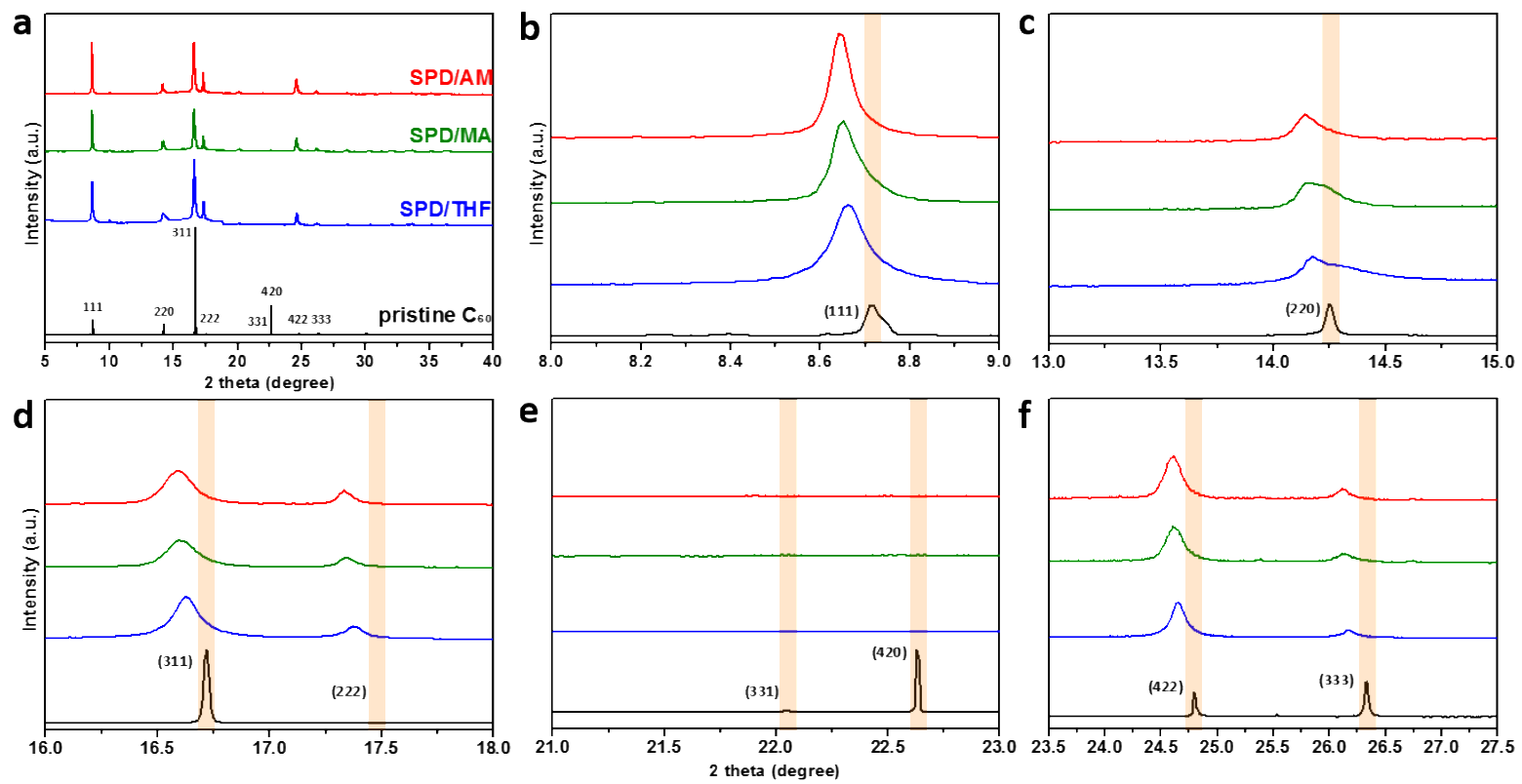

Figure S8. X-ray diffraction patterns for pristine $\mathrm{C}_{60}$ (black), $\mathrm{K}_{3} \mathrm{C}_{60}$ prepared by $\mathrm{SPD}$ methods, SPD/AM (red), SPD/MA (green) and SPD/THF (blue), respectively. (a) (f) XRD patterns for 2 theta scan range (a) from $5.0^{\circ}$ to $40.0^{\circ}$, (b) from $8.0^{\circ}$ to $9.0^{\circ}$, (c) from $13.0^{\circ}$ to $15.0^{\circ}$, (d) from $16.0^{\circ}$ to $18.0^{\circ}$, (e) from $21.0^{\circ}$ to $23.0^{\circ}$, (f) from $23.5^{\circ}$ to $27.5^{\circ}$. 
Table S1. Spin amount determination of potassium added $\mathrm{C}_{60}$ solutions. The used $\mathrm{C}_{60}$ is equal to $12.5 \mathrm{mg}\left(1.73 \times 10^{-2} \mathrm{mmol}\right)$ each. The DI/ $\mathrm{N}$ value is the double integrated (DI) intensity of the EPR spectra that have been normalized $(\mathrm{N})$ to account for conversion time, receiver gain, number of data points and sweep width.

\begin{tabular}{ccc}
\hline Sample & Used K amount (mg, mmol) & DI/N \\
\hline $\mathrm{K}_{3} \mathrm{C}_{60}$ & $2.03 \mathrm{mg}, 5.20 \times 10^{-2} \mathrm{mmol}$ & 3607 \\
$\mathrm{~K}_{2} \mathrm{C}_{60}$ & $1.36 \mathrm{mg}, 3.47 \times 10^{-2} \mathrm{mmol}$ & 2615 \\
$\mathrm{~K}_{1} \mathrm{C}_{60}$ & $0.68 \mathrm{mg}, 1.73 \times 10^{-2} \mathrm{mmol}$ & 1245 \\
$\mathrm{~K}_{0} \mathrm{C}_{60}$ & - & - \\
\hline
\end{tabular}


Table S2. Shielding fraction of solution-phase-doped $\mathrm{K}_{3} \mathrm{C}_{60}$ depending on method and annealing time.

\begin{tabular}{|c|c|c|c|}
\hline annealing time method & SPD/AM & SPD/MA & SPD/THF \\
\hline 24 & $54.56 \%$ & $61.18 \%$ & $2.01 \%$ \\
\hline 30 & $89.54 \%$ & $88.58 \%$ & $31.07 \%$ \\
\hline 36 & $87.93 \%$ & $60.37 \%$ & $22.80 \%$ \\
\hline 42 & $62.40 \%$ & $76.47 \%$ & $87.05 \%$ \\
\hline 48 & $61.52 \%$ & $79.72 \%$ & $35.92 \%$ \\
\hline
\end{tabular}


Table S3. Assigned crystal information of $\mathrm{K}_{3} \mathrm{C}_{60}$.

\begin{tabular}{|c|c|c|c|}
\hline sample & crystal system & a $(\AA ̊)$ & $\begin{array}{l}\text { volume per } \\
\text { unit cell }\left(\AA^{3}\right)\end{array}$ \\
\hline $\mathrm{K}_{3} \mathrm{C}_{60}$ (SPD/THF) & cubic & $14.245(3)$ & $2890.74(7)$ \\
\hline $\mathrm{K}_{3} \mathrm{C}_{60}(\mathrm{SPD} / \mathrm{MA})$ & cubic & $14.245(2)$ & $2891.05(6)$ \\
\hline $\mathrm{K}_{3} \mathrm{C}_{60}$ (SPD/AM) & cubic & $14.247(1)$ & $2892.21(3)$ \\
\hline pristine $\mathrm{C}_{60}$ & cubic & $14.135(2)$ & $2825.57(5)$ \\
\hline
\end{tabular}

